\title{
Assistência da enfermagem aos pacientes traqueostomizados internados em um hospital de médio porte do Vale do Taquari-RS-Brasil
}

\author{
Nursing care for tracheostomized patients admitted to a medium-sized hospital in Vale do Taquari-
} RS-Brazil

Atención de enfermería a pacientes traqueostomizados ingresados en un hospital de tamaño mediano en Vale do Taquari-RS-Brasil

\section{Resumo}

Objetiva-se verificar o conhecimento de como é a assistência da enfermagem aos pacientes traqueostomizados internados em um Hospital de Médio Porte do Vale do Taquari-RS-Brasil. Trata-se de uma metodologia descritiva, qualitativa. A coleta de dados se deu por meio de entrevista com questões norteadoras com 10 enfermeiros do Hospital de Médio Porte do Vale do Taquari, que realizam assistência e aceitaram responder a entrevista, e assinaram o Termo de Livre e Esclarecido (TCLE). Foram excluídos enfermeiros que estavam afastados de suas atividades. As respostas foram analisadas de acordo com o conteúdo de Bardin, e organizadas em quatro categorias. Observa-se corretamente a devida assistência do enfermeiro aos pacientes traqueostomizados internados no Hospital de Médio Porte do Vale do Taquari-RS-Brasil, conclui-se que os participantes conhecem a assistência correta e seus respectivos cuidados.

Palavras-chave: Traqueostomia; Assistência integral à saúde; Enfermeiros.

\begin{abstract}
The objective of this study is to verify the knowledge of nursing care for tracheostomized patients hospitalized in a Medium-sized Hospital of the Taquari Valley-RS-Brazil. It is a descriptive, qualitative methodology. Data collection took place through interviews with guiding questions to nurses at the Medium-sized Hospital of Vale do Taquari. The study included 10 nurses who provide assistance and who accepted to answer the interview. The exclusion criterion was for nurses who were away from their activities. All study participants signed the Free and Informed Consent Form (ICF). The responses were analyzed and collected according to the content of Bardin (2011), and organized into four categories. From the results obtained, the proper assistance of the nurse to the tracheostomized patient is correctly observed. Through assistance to tracheostomized patients admitted to the Medium-sized Hospital of Vale do TaquariRS-Brazil, it is concluded that the participants know the correct care and their respective care.
\end{abstract}

Keywords: Tracheostomy; Integral health assistance; Nurses.

\section{Resumen}

El objetivo de este estudio es verificar el conocimiento de la atención de enfermería para pacientes traqueostomizados hospitalizados en un Hospital mediano del Valle de Taquari-RS-Brasil. Es una metodología descriptiva y cualitativa. La recolección de datos se llevó a cabo a través de entrevistas con preguntas orientadoras a enfermeras del Hospital Mediano de Vale do Taquari. El estudio incluyó a 10 enfermeras que brindan asistencia y que aceptaron responder a la entrevista. El criterio de exclusión fue para enfermeras que se encontraban alejadas de sus actividades. Todos los participantes del estudio firmaron el formulario de consentimiento libre e informado (ICF). Las respuestas se analizaron y recopilaron según el contenido de Bardin (2011), y se organizaron en cuatro categorías. A partir de los resultados obtenidos se observa correctamente la adecuada asistencia de la enfermera al paciente traqueostomizado. A través de la 
asistencia a los pacientes traqueostomizados ingresados en el Hospital Mediano de Vale do Taquari-RS-Brasil, se concluye que los participantes conocen la atención correcta y su respectiva atención.

Palabras clave: Traqueostomía; Asistencia integral a la salud; Enfermeros.

\section{Introdução}

A respiração é um processo que acontece com a colaboração de vários órgãos, definido como sistema respiratório. Entretanto, se houver interrupção da respiração, a traqueostomia será realizada para que haja medida de sobrevivência. A abertura da traqueia para o acesso da via aérea superior é um método cirúrgico, podendo ser um procedimento eletivo ou de urgência, e tem a definição de traqueostomia (Rodrigues Filho, \& Junges, 2017).

Historicamente, a traqueostomia era realizada em pessoas com traumas e vias respiratórias bloqueadas, provocadas na guerra. O ressurgimento da traqueostomia voltou no século XIX, assim como tubos com melhores adaptações e mais flexíveis. O surgimento de avanços técnicos, só favoreceu o procedimento da traqueostomia, o desenvolvimento da traqueostomia foi para promover a desobstrução das vias aéreas, facilitando a respiração (França, Takimoto, \& Cervantes, 2017).

A traqueostomia finalmente conquistou seu espaço nos pacientes críticos com suporte ventilatório pela década de 60, pelo surgimento das unidades de terapias intensivas e ventiladores de pressão positiva, pois antes havia sido desconsiderada por diversas vezes a sua utilização (Sakae, Sakae, Schmitz, \& Sakae, 2015).

A garantia da qualidade no procedimento conta com cuidados em todas as suas fases, mas, principalmente, nos cuidados pós-operatórios a que a equipe de enfermagem possui um papel fundamental, oferecendo a assistência correta para que não ocorram complicações (Andrade, 2000).

Algumas consequências podem ocorrer quando há falta de treinamento da equipe e falta de conhecimento, pois a prioridade sempre será a prevenção, bem-estar do paciente e redução no tempo de internação. A conduta profissional será sempre fundamental para a recuperação do paciente (Souza, Alves, Santana, 2016).

Desta forma, durante a pesquisa, se buscou coletar e relacionar os dados baseados no relato de enfermeiros, onde a pesquisa possuiu por objetivo verificar o conhecimento de como é a assistência da enfermagem aos pacientes traqueostomizados internados em um Hospital de Médio Porte do Vale do Taquari-RS-Brasil.

\section{Metodologia}

Estudo exploratório e descritivo, com abordagem qualitativa, desenvolvido por uma estudante do curso de Enfermagem, durante a realização do seu trabalho de conclusão de curso. Os dados foram analisados por meio da análise de conteúdo de Bardin (2011). A amostra foi composta por 10 enfermeiros do Hospital de Médio Porte do Vale do Taquari-RS-Brasil que aceitaram responder a entrevista.

Os critérios de inclusão para os participantes foram enfermeiros que realizam assistência no hospital e que aceitaram ser entrevistados. Os critérios de exclusão foram definidos a todos os profissionais que estavam em períodos de férias, licença maternidade ou licença saúde e enfermeiros que se recusaram a participar da pesquisa.

Inicialmente, os pesquisadores entraram em contato com a coordenação do setor que realizou o mapeamento dos profissionais e, após, definido os participantes e contatados individualmente, sendo apresentado o projeto, bem como seus objetivos e critérios éticos envolvidos. Foi realizado o convite para a participação; após o aceite, foi agendada entrevista individual com cada participante.

A aplicação da entrevista foi realizada no referido Hospital nos turnos manhã, tarde e noite, com os profissionais que encontravam-se em assistência e ocorreram em espaço cedido pela instituição hospitalar. Para o registro das entrevistas foi utilizado um gravador de áudio, com duração em média de 20 minutos.

A entrevista foi realizada com base em cinco questões norteadoras. Sendo elas transformadas em quatro categorias: 1) 
Conhecimento sobre os cuidados que a equipe de enfermagem presta aos pacientes traqueostomizados internados; 2) Procedimentos realizados pelos enfermeiros aos pacientes traqueostomizados internados; 3 ) Principais dificuldades encontradas na assistência aos pacientes traqueostomizados internados; e 4) Habilidades e competências exigidas dos enfermeiros no cuidado aos pacientes traqueostomizados.

As informações coletadas foram transcritas, agrupadas e analisadas conforme a análise de conteúdo de Bardin (2011).

A pesquisa respeitou a Resolução 466/12 do Conselho Nacional de Saúde que trata da pesquisa envolvendo seres humanos

\section{Resultados e Discussão}

\subsection{Conhecimento sobre os cuidados que a equipe de enfermagem presta aos pacientes traqueostomizados internados}

A traqueostomia é a principal indicação em pacientes que recebem suporte ventilatório prolongado, pois estão expostos a complicações tardias por estar com intubação endotraqueal prolongada. Possui benefícios para o paciente, como melhor conforto, manuseio fácil para equipe de enfermagem, melhora na higiene oral, possibilidade de uma melhora na ingestão oral, comunicação e menor taxa de autoextubação da traqueostomia (Sakae et al., 2015).

A maioria dos entrevistados respondeu que os cuidados prestados pela equipe de enfermagem aos pacientes traqueostomizados são a realização de aspiração, monitorização da posição, trocas de curativos e identificação de sinais de infecções.

Deve-se realizar aspiração da traqueo e vias aéreas no mínimo uma vez ao turno ou sempre que se fizer necessário. Monitorar a posição do paciente, facilitando a respiração, assim como troca de curativo (Enf.1).

[...] realização de aspiração da traqueo, assim como troca de curativos [...] (Enf. 2).

O cuidado com os pacientes é essencial, a aspiração traqueobrônquica é um procedimento invasivo, realizado para remover secreções e favorecer a melhora na qualidade das vias aéreas (Souza, Alves, \& Santana, 2016).

\subsection{Procedimentos realizados pelos enfermeiros aos pacientes traqueostomizados internados}

Em relação aos procedimentos realizados pelos enfermeiros no hospital, se observa o cuidado com o curativo o mais citado, e a importância de realizar uma aspiração correta. Então, sobre os procedimentos que os enfermeiros realizam no hospital, foram citados:

[...] Aspiração traqueal com material estéril, higienização da endocânula e monitorização dos sinais vitais. Evitar que a traqueostomia não seja tracionada, pois pode causar lesões de traqueia (Enf.5).

Aspiração em orifício endocânula, auxiliar o médico para a troca da endocânula por metálica. Realizar curativo ou supervisionar uma vez ao turno (Enf 8).

Por ser um procedimento invasivo para remover secreções e favorecer a melhora na qualidade das vias aéreas e ventilação mecânica, a indicação é para pacientes com dificuldade em tossir ou expelir o acúmulo de secreção naturalmente, principalmente em pacientes pós-operatórios, confusos mentalmente, com tubo endotraqueal ou traqueostomizados (Souza, Alves, \& Santana, 2016). 


\subsection{Principais dificuldades encontradas na assistência aos pacientes traqueostomizados internados}

Foram abordadas pelos entrevistados quais as principais dificuldades na assistência. A traqueostomia, apesar de promover enormes benefícios ao paciente, também poderá causar algumas complicações, pois o enfermeiro precisa saber a técnica e os cuidados corretos. O enfermeiro possui o papel de orientar o paciente e seus familiares sobre o devido cuidado a ser tomado (HC-UFTM, 2018).

Por ser um cuidado de técnica e autocontrole, os enfermeiros relatam a principal dificuldade na assistência ao paciente traqueostomizado, sendo a comunicação com o mesmo, vivenciado dentro do hospital.

Uma das principais dificuldades é a comunicação entre o profissional e o paciente, pois sem a comunicação o cuidado não será eficaz (Enf. 3).

Comunicação, entender e orientar o paciente sobre os benefícios da traqueo e a sua importância para a recuperação dele (Enf. 2).

\subsection{Habilidades e competências exigidas dos enfermeiros no cuidado aos pacientes traqueostomizados}

Cuidados com a traqueostomia são importantes para o paciente, como a realização da limpeza da subcânula no mínimo três vezes ao dia é uma segurança nos casos de obstrução. Deve-se manter uma boa higienização ao redor da traqueostomia, limpando com água e sabonete de glicerina após fazer hidratação da pele. Utilizar duas gazes dobradas mantendo entre a cânula e o pescoço do paciente, sempre limpo e seco, evitando que aconteçam irritações na pele ao redor da traqueostomia (Brasil, 2015).

A assistência no pós-operatório exige realizar cuidados pós-extubação, a observação constante do paciente feita pela equipe de enfermagem possui nesse momento uma grande importância. Verificação da frequência respiratória, saturação do oxigênio, dispneia e observação se nas extremidades há presença de cianose (Góes, Silva, \& Lima, 2017).

O enfermeiro possui algumas ações privativas, como controlar e fazer a prevenção de infecções, controlar dados relacionados à assistência, cuidados com pacientes graves e cuidados que exigem seu conhecimento científico, assim como tomadas de decisões rápidas e passar seus conhecimentos para sua equipe (Souza, Alves, \& Santana, 2016).

[...] Saber as técnicas de aspiração, orientações aos familiares, verificação do balonete, monitorização de sinais e sons vinso da traqueo e higienização e troca da endocânula (Enf 3).

[...] Conhecimento científico e capacidade de tomar decisões imediatas. Assegurar a assistência adequada e sempre estar em alerta se algum dos sinais vitais estiver alterado (Enf 5).

A partir dos resultados obtidos, se observa a importância da assistência aos pacientes traqueostomizados durante sua internação, cuidados e procedimentos a serem realizados com técnica asséptica.

Um procedimento de traqueostomia com garantia de qualidade está principalmente ligado ao pós-operatório, sendo que a equipe de enfermagem possui um papel de grande importância, com sua assistência correta, para que não aconteça futuras complicações ao paciente (Andrade, 2000).

O controle e a prevenção são ações privativas do enfermeiro, que exigem conhecimento científico, atuando não só no cuidado em procedimentos realizados, função do enfermeiro assistencial, mas também na gestão e educação, oferecendo uma melhora e assistência na qualidade de vida desses pacientes (Souza, Alves, \& Santana, 2016).

Um modelo que poderá ser seguido nos cuidados aos pacientes é a Sistematização da Assistência de Enfermagem (SAE). O cuidado da enfermagem com a aplicação da SAE se torna científico, é realizada uma assistência humanizada, diagnosticada, 
intervindo e classificando para que as ações sejam organizadas com eficiência (Pereira, 2014).

Instruída sempre pelo enfermeiro, a prática do cuidado realizada pela equipe de enfermagem contribui no suporte do cuidado, para que aconteça de uma forma segura e sistematizada. As resoluções estabelecidas no código de ética descrevem a constituição da equipe mínima, importância do pessoal, registro em prontuário do paciente e a utilização do manual de procedimentos padronizados e suas rotinas (Andrade, Piccoli, RuoffI, Ribeiro, \& Sousa, 2016).

Nas condutas realizadas pelos enfermeiros do hospital em relação a assistência ao paciente traqueostomizado, são satisfatórias, pois conseguem exercer a devida função no cuidado a esses pacientes. Percebe-se a importância de possuir as habilidades e orientações passadas a eles.

\section{Considerações Finais}

Este estudo buscou conhecer a assistência do enfermeiro ao paciente traqueostomizado internado em um Hospital de Médio Porte do Vale do Taquari-RS, tendo como objetivo saber se essa assistência está sendo abordada e desempenhada perante a equipe de enfermagem.

Quanto aos enfermeiros entrevistados, conseguimos perceber que cada um efetua seu papel e forma de trabalhar com o paciente de modo adequado, havendo uma boa comunicação na orientação e habilidades no cuidado, assim como nos procedimentos que são obrigatórios saber sua procedência e como realizá-los. Podemos perceber, por meio das entrevistas, a importância em verificar os sinais vitais, a forma correta de realizar uma aspiração, cuidados com o curativo, para que não haja infecções, e a comunicação do enfermeiro na maneira que ele orienta o paciente e seus familiares.

Conclui-se que a assistência de enfermagem aos pacientes traqueostomizados está de acordo para a contribuição do cuidado ao mesmo. O estudo demonstra a realidade da assistência no hospital ao paciente traqueostomizado e sua importância em ser realizado de uma forma correta.

\section{Referências}

Andrade, M. T. S. (2000). Isolamento das vias respiratórias: a traqueostomia. In: Andrade, M. T. S. Cuidados intensivos. Rio de Janeiro: Mc Graw-hill Interamericana do Brasil Ltda. . 219-251.

Andrade, S. R., Piccoli, T., RuoffI, A. B., Ribeiro, J. C., \& Sousa, F. M. (2016). Fundamentos normativos para a prática do cuidado realizado pela enfermagem brasileira. Revista Brasileira de Enfermagem, 69(6),1082-1090. 10.1590/0034-7167-2016-0228

Bardin, L. (2011). Análise de conteúdo. (2a ed.): Edições 70.

França, P. D. S., Takimoto, R. M., \& Cervantes, O. (2017). Cirurgia de cabeça e pescoço: via aérea cirúrgica. In: Abrahão, M., \& Neves, L. R. Emergências e urgências em otorrinolaringologia e cirurgia de cabeça e pescoço: Atheneu. p. 3-573.

Góes, R. S. S., Silva, S. O. P., \& Lima, C. B. (2017). Traqueostomia na Unidade de Terapia Intensiva: visão do enfermeiro. Temas em Saúde, 17(4), 228-241. http://temasemsaude.com/wp-content/uploads/2018/01/17416.pdf

Hospital de Clínicas da Universidade Federal do Triângulo Mineiro (HC-UFTM). (2018).

POP: Traqueostomia: Cuidados e Decanulação: unidade de reabilitação: Ebserh/Ministério da Educação.

Pereira, A. C. O. (2014). Sistematização da assistência de enfermagem no atendimento da urgência do sangramento varicoso (Especialização). Universidade Federal de Santa Catarina - UFSC, Florianópolis, SC, Brasil.

Rodrigues Filho, E. M., \& Junges, J. R. (2017). Traqueostomia no doente crítico na era do consentimento livre e esclarecido. Revista Bioética, 25(3), 502-511. $10.1590 / 1983-80422017253207$

Sakae, T. M., Sakae, G. R. F. M, Schmitz, R. L., \& Sakae, D. Y. (2015). Comparação da mortalidade para traqueostomia precoce e tardia em pacientes cardiológicos de uma unidade de terapia intensiva no Sul do Brasil. Arquivos Catarinenses de Medicina, 45(1), 3-12. http://www.acm.org.br/acm/seer/index.php/arquivos/article/view/57

Souza, L. C. A., Alves, R. M., \& Santana, C. J. (2016). Atribuições do enfermeiro na prevenção da pneumonia associada a ventilação mecânica na unidade de terapia intensiva. Revista Uningá Review, 26(1), 63-67. http://revista.uninga.br/index.php/uningareviews/article/view/1786 